Aims To use gender ratio to detect greater than expected numbers of gastrointestinal infections in MSM.

Methods We examined annual male to female ratios of laboratory confirmed patient-episodes from those aged 16-65 years with no known history of travel for eight gastrointestinal pathogens (Campylobacter, Cryptosporidium, Giardia, Hepatitis A, Norovirus, Salmonella, Shigella, and VTEC) in England between 2003 and 2013. Chi-squared tests for linear trend were conducted and a male to female ratio of more than two was considered suggestive of an excess. Sub-analyses by age and high-risk areas (London, Brighton and Manchester) were conducted.

Results An increased linear trend and excess of male episodes was observed for Shigella ( $\mathrm{p}<0.001$; m:f ratio of 2.0 and 2.5 in 2012 and 2013, respectively) but not the other gastrointestinal infections. Consistent with MSM-mediated transmission, the excess of male Shigella episodes was most pronounced among those aged 25-49 years (ratios of 2.4 and 2.9) and those in high-risk areas (ratios of 2.9 and 4.0); no excess was observed among children.

Conclusion This method identified the recent outbreak of Shigella and routine application might alert public health authorities to some future gastrointestinal infection outbreaks in MSM. Utility of this approach to detect excess episodes among MSM is likely to be pathogen specific and dependent on several factors including R0.

\section{P217 HIGH DEMAND FOR AN MSM CLINIC PILOT IN A DISTRICT GENERAL HOSPITAL}

${ }^{1,2}$ Mamatha Oduru*, ${ }^{2}$ Emma Wainwright, ${ }^{2}$ Janice Burnett, ${ }^{2}$ Alan Tang. ${ }^{1}$ The Garden Clinic, Slough, UK; ${ }^{2}$ The Florey Unit, Reading, UK

10.1136/sextrans-2015-052126.261

Background/introduction A tailored sexual health service for men who have sex with men (MSM) was piloted in a district general hospital. This was in response to a 'Gay Pride' survey in which $80 \%$ of surveyed attendees requested a specialised local service.

Aim(s)/objectives This clinic explored the feasibility and acceptability of a targeted MSM service in the district general hospital setting.

Methods The pilot had 9 clinics over 3 months. Each patient was fast-tracked to a multidisciplinary team (doctor, nurse and health advisor). A rapid HIV test (result within $24 \mathrm{~h}$ ) was offered to every patient as point of care testing was unavailable in the service. Data and patient feedback were analysed in SPSS version 22.

Results A total of 13 patients attended the clinic (new, $\mathrm{N}=12$, follow up, $\mathrm{N}=1$ ). Age range was 22 to 67 years old (mean 33.7, standard deviation 13.27) and all were Caucasian. 6/13 attended for an asymptomatic screen; the prevalence of sexually transmitted infections (STI's) was 46\%. All tested HIV negative. $61.5 \%$ had no prior immunity to Hepatitis B. $92.3 \%$ considered the rapid HIV test an incentive to attend and 100\% wanted a future point of care test. There was favourable patient feedback.

Discussion/conclusion The clinic pilot was positively received and the majority rated it preferable to the routine genitourinary clinic. There was a high prevalence of STI's and high rate of opportunistic Hepatitis B vaccination. There is a notable demand for rapid HIV testing. Our tailored MSM clinic encouraged attendance in a high risk provincial population.
P218 RISING STI RATE IN FEMALE SEX WORKERS ATTENDING AN INNER CITY DEDICATED CLINIC

${ }^{1}$ Elizabeth Williams, ${ }^{2}$ Kim Leverett*, ${ }^{1}$ Vanessa Apea. ${ }^{1}$ Ambrose King Centre, Barts Health NHS Trust, London, UK; ${ }^{2}$ Homerton University Hospital NHS Trust, London, UK

\subsection{6/sextrans-2015-052126.262}

Background/introduction Our GUM service has a dedicated sex worker (SW) clinic. In 2014 there was an anecdotal increase in in CT and GC diagnoses in patients attending this clinic therefore a retrospective case note review was performed to assess this observation and explore causal factors.

Aim(s)/objectives To compare number of CT/GC infections in the SW clinic in 2012 and 2014.

Methods Notes were reviewed for age, ethnicity and CT/GC codes in all patients seen in 2012 and 2014. The notes of those found to be CT or GC positive had a more detailed review for type of services offered, condom use, place of work (i.e. flat, sauna). Chi-squared test was used to calculate $\mathrm{p}$ value.

Results

\begin{tabular}{llll} 
Abstract P218 Table 1 & \multicolumn{4}{l}{ STI rates in female sex workers } & \\
\hline Year & 2012 & 2014 & P value \\
\hline Number of patients: & 192 & 140 & \\
- Attending & $19-58$ & $19-54$ & \\
- Age Range & $5 / 192(2.6 \%)$ & $19 / 140(13.6 \%)$ & 0.0002 \\
- With either CT or GC at least once & 0 & $3 / 140(2.1 \%)$ & \\
- With both CT and GC concurrently & 0 & $3 / 140(2.1 \%)$ & \\
- That had repeat infections in the same year & 0 & 16 & \\
Total number of CT infections & 4 & 11 & \\
Total number of GC infections & 1 & &
\end{tabular}

Discussion/conclusion There was a significant increase in STIs from 2012 to 2014. In addition, concurrent CT and GC infection and repeat infections were seen in 2014, but not in 2012. This observation has guided an update in patient education materials and a more targeted approach to outreach. Possible infection clusters are being explored.

\section{P219 DOES A SPECIALISED MSM CLINIC IN A DISTRICT GENERAL HOSPITAL ATTRACT A RISKIER POPULATION?}

1,2 Mamatha Oduru ${ }^{*},{ }^{2}$ Emma Wainwright, ${ }^{2}$ Janice Burnett, ${ }^{2}$ Alan Tang. ${ }^{1}$ The Garden Clinic, Slough, UK; ${ }^{2}$ The Florey Unit, Reading, UK

10.1136/sextrans-2015-052126.263

Background/introduction A sexual health service for men who have sex with men (MSM) was piloted due to local demand for a specialised clinic with an understanding of MSM risk taking behaviour and sexual practices.

Aim(s)/objectives To explore if an MSM clinic in a district general hospital attracts a patient population with riskier sexual behaviour compared to the normal genitourinary (GU) service. Methods Risk data was collected from all MSM patients attending the specialised and routine GU clinics over a 3 month period using self-completed questionnaires. 'High risk' behaviour was classified as any of the following within the past 3 months: group sex, sex parties, chem-sex, casual partner sourcing online, sexual activity in London and number of partners. Data were analysed in SPSS version 22.

Results Total sample size was 40 (MSM clinic, $\mathrm{N}=13$, GU clinic, $\mathrm{N}=27$ ). Age range was 18 to 67 years old (mean 32.47, 